\title{
Interval-Valued Hidden Markov Models for Recognizing Personality Traits in Social Exchanges in Open Multiagent Systems
}

\author{
G.P. DIMURO, A.C.R. COSTA, L.V. GONÇALVES, A. HÜBNER ${ }^{1}$ PPGINF, UCPel, \\ 96010-000 Pelotas, RS, Brazil.
}

\begin{abstract}
This paper presents an application of Interval-valued Hidden Markov Models to the modelling of agent personality traits in multiagent systems. The agents' behaviors are modeled as probabilistic transitions functions, where interval-valued probabilities are used to express the uncertainty in determining those probabilities. The model of regulation of social exchanges is based on the concept of equilibrium supervisor, which is able to recommend the best exchanges for the agents to perform in order to achieve the equilibrium of the system.
\end{abstract}

Key-words. Interval Mathematics, Hidden Markov Models, Social Exchanges Values, Multiagent Systems

\section{Introduction}

This work is concerned with the use of Interval Mathematics [7] and Interval-valued Hidden Markov Models [11] in models for Social Simulation in multiagent systems [14].

Social control is a powerful notion for explaining the self-regulation of a society, and the various possibilities for its realization have been considered, both in natural and artificial societies [1]. The approach for regulating interactions in multiagent systems, based on the Piaget's theory of social exchange values [8], was proposed in [5]. The evaluation of an exchange by an agent is done on the basis of a scale of exchange values, which are of a qualitative nature - subjective values like those everyone uses to judge the daily exchanges (good, bad etc.). In order to capture the qualitative nature of exchange values, techniques from Interval Mathematics were used. A scale of interval exchange values was defined as an algebraic structure endowed with a loose equivalence relation - two intervals are equivalent if their midpoints are approximately equal (see [5]).

The social exchange control mechanism is performed by a supervisor agent, which can solve the problem of keeping agent interactions in equilibrium (with respect to the exchange values involved in them) by using Qualitative Interval Markov Decision Processes (QIMDP) [3] - MDPs [9] where states are classes of balances of exchange values, actions are interval operations, and the equilibrium state is the class of intervals enclosing the zero.

As explained in [2], a realistic account of agent interactions has to consider that agents may have different interaction personalities, in order to allow for the agents to participate

\footnotetext{
${ }^{1}$ e-mail: $\{$ liz,rocha,llvarga,hubner $\} @$ ucpel.tche.br
} 
in different ways in social interactions, depending not only on the way tasks were delegated to them, but also on the way they assess their own contributions and the contributions of the others to the interaction. Here, the agents may have different personality traits, which induce different attitudes towards the regulation mechanism (blind obedience, eventual obedience etc.) and the possible profits of social exchanges (egoism, altruism etc.). Also, these personality traits influence the agents' evaluation of their current status (realism, over- or under-evaluation). These variables create a probabilistic social environment, from the point of view of the social control.

In [4], we consider that the agents allow full external access to their internal states, behaving as transparent agents. So, the supervisor is always able to determine the status of the system, acting in a completely observable environment. This paper considers partially observable environments, i.e., open societies where, at each instant, new agents may joint the society, appearing as non-transparent agents, which means that the supervisor has no direct knowledge of the new agents' personality traits and no direct access to their internal values. Thus, it must rely on observations of what the agents report about the exchanges.

The personality traits are defined by probability distributions that reflect the exchanges performed by the agents in each interaction. In this paper, we extend previous work [4] to consider interval-valued probabilities [13] that characterize the uncertainty in the modelling of the different personality-based behaviors.

To solve the problems of determining the most probable current state of the system, recognizing agent's personalities, learning new personalities traits, and maintaining an adequate model of the system, we introduce into the supervisor a mechanism based on Interval-valued Hidden Markov Models (I-HHM), using a generalization of HMM [10] to consider interval-valued probabilities first introduced in [11].

The paper is organized as follows. In Sect. 2., we review the modelling of social exchanges. Section 3. presents the architecture of the regulation mechanism. Section 4. introduces the exchanges between personality-based agents. The I-HMM is introduced in Sect. 5., and simulation results in Sect. 6.. Section 7. is the Conclusion.

\section{Modelling Social Exchanges}

The evaluation of an exchange by an agent is done using a scale of exchange values, which are represented as intervals $X=\left[x_{1}, x_{2}\right]$, with $-L \leq x_{1} \leq x \leq x_{2} \leq L, x_{1}, x_{2}, L \in \mathbb{R}$. This representation is a compromise between a purely qualitative and a purely quantitative representation. It makes the representation mathematically operational, and the decision process computationally viable, without being unfaithful to Piaget's approach [8].

A social exchange between two agents, $\alpha$ and $\beta$, is performed involving two types of stages. In stages of type $\mathrm{I}_{\alpha \beta}$, the agent $\alpha$ realizes a service for $\beta$. The exchange values involved in this stage are the following: $r_{\mathrm{I}_{\alpha \beta}}$ (the value of the investment done by $\alpha$ for the realization of a service for $\beta$, which is always negative); $s_{\mathrm{I}_{\beta \alpha}}$ (the value of $\beta$ 's satisfaction due to the receiving of the service done by $\alpha$ ); $t_{\mathrm{I}_{\beta \alpha}}$ (the value of $\beta$ 's debt, the debt it acquired to $\alpha$ for its satisfaction with the service done by $\alpha$ ); and $v_{\mathrm{I}_{\alpha \beta}}$ (the value of the credit that $\alpha$ acquires from $\beta$ for having realized the service). In stages of the type $\mathrm{II}_{\alpha \beta}$, the agent $\alpha$ asks the payment for the service previously done for $\beta$, and the values related with this exchange have similar meaning. $r_{\mathrm{I}_{\alpha \beta}}, s_{\mathrm{I}_{\beta \alpha}}, r_{\mathrm{II}_{\beta \alpha}}$ and $s_{\mathrm{II}_{\alpha \beta}}$ are called material 
values. $t_{\mathrm{I}_{\beta \alpha}}, v_{\mathrm{I}_{\alpha \beta}}, t_{\mathrm{II}_{\beta \alpha}}$ and $v_{\mathrm{II}_{\alpha \beta}}$ are the virtual values. The order in which the exchange stages may occur is not necessarily $\mathrm{I}_{\alpha \beta}-\mathrm{II}_{\alpha \beta}$. We observe that the values are undefined if either no service is done in a stage of type I, or no credit is charged in a stage of type II. Also, it is not possible for $\alpha$ to realize a service for $\beta$ and, at the same, to charge him a credit. Observe that, in any exchange stage, either $\alpha$ or $\beta$ has to perform a service, so decreasing its material results.

A social exchange process is a sequence of stages of type $\mathrm{I}_{\alpha \beta}$ and/or $\mathrm{II}_{\alpha \beta}$. The material results, according to the points of view of $\alpha$ and $\beta$, are given by the sum of the well defined material values involved in the process, denoted, respectively, by $\mathbf{m}_{\alpha \beta}$ and $\mathbf{m}_{\beta \alpha}$. The virtual results $\mathbf{v}_{\alpha \beta}$ and $\mathbf{v}_{\beta \alpha}$ are defined analogously. A social exchange process is said to be in equilibrium if $\mathbf{m}_{\alpha \beta}$ and $\mathbf{m}_{\beta \alpha}$ are around a reference value $s \in \mathbb{R}$.

\section{The Regulation Mechanism}

Figure 1 shows the architecture of our social exchange regulation mechanism, which extends the one proposed in [4] with a learning module based on I-HMMs. The equilibrium supervisor, at each time, uses an Evaluation Module to analyze the constraints imposed by the system's external and internal environments, determining the target equilibrium point $s$. To regulate transparent agents, the supervisor uses a Balance Module (Sum.) to calculate their balances of material/virtual results of the performed exchanges. To regulate nontransparent agents, the supervisor uses an observation module (Obs.) to access what they report about their virtual values (debts/credits), and the I-HMM module to recognize and maintain an adequate model of the personality traits of such agents, generating plausible balances of their material exchange values.

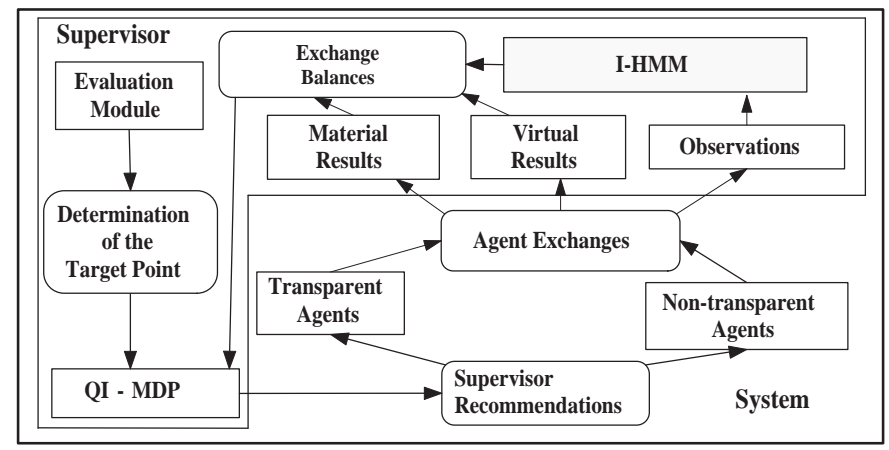

Figure 1: The architecture of the social exchange regulation mechanism

Taking both the directly observed and the indirectly calculated material results, together with the currently target equilibrium point, the supervisor uses the module that implements a personality-based QI-MDP to decide on recommendations of exchanges for the agents, in order to keep the material results in equilibrium. It also takes into account the virtual results in order to decide which type of exchange stage it should suggest.

The states of a QI-MDP [3] are pairs $\left(E_{\alpha, \beta}, E_{\beta, \alpha}\right)$ of classes of material results (investments and satisfactions) of exchanges between agents $\alpha$ and $\beta$, from the point of view 
of $\alpha$ and $\beta$, respectively. ${ }^{2} \mathbf{E}_{s}=\left\{E_{s}^{-}, E_{s}^{0}, E_{s}^{+}\right\}$is the set of the supervisor representation of the classes of unfavorable $\left(E_{s}^{-}\right)$, equilibrated $\left(E_{s}^{0}\right)$ and favorable $\left(E_{s}^{+}\right)$material results of exchanges, related to a target equilibrium point $s .\left(E_{\alpha, \beta}^{0}, E_{\beta, \alpha}^{0}\right)$ is the terminal state (equilibrium state).

The actions of the QI-MDP model are interval operations that give rise to state transitions. The actions may be of the following types: a compensation action, which directs the agents' exchanges to the equilibrium point; a go-forward action, which directs them to increasing material results; a go-backward action, which directs them to decreasing material results. The supervisor has to find, for the current state, the actions that may achieve the terminal state in the least number of steps, which is called an optimal police. The choice of actions is constrained by the rules of the social exchanges and some transitions are forbidden (e.g., both agents increasing results simultaneously), so in some cases the supervisor has to find alternative paths in order to lead the system to the equilibrium.

An optimal police generates an optimal exchange recommendation, which is a partially defined exchange stage that the agents are suggested to perform. Also, by the analysis of the agents' virtual results (debts/credits), the supervisor recommends a specific type of exchange stage (I or II).

\section{Personality-based Agents}

We define different levels of obedience to the supervisor that the agents may present:

Blind Obedience: the agent always follows the recommendations;

Eventual Obedience: the agents may not follow the recommendations, according to a certain probability;

Full Disregard of Recommendations: the agent always decides on its own, disregarding what was recommended.

The agents may have different social attitudes that give rise to an interval state-transition function, which specify, for each obedience level, and given the current state and recommendation, an interval-valued probability distribution $\Pi\left(\mathbf{E}_{s}\right)$ over the set of states $\mathbf{E}_{s}$ that the interacting agents will try to achieve next, depending on the their personality traits. In the following, we illustrate some of those personality traits:

Egoism: the agent is mostly seeking its own benefit, with a high probability to accept exchanges that represent transitions toward states of favorable results;

Altruism: the agent is mostly seeking the benefit of the other, with a high probability to accept exchanges that represent transitions to states where the other has favorable results;

Fanaticism: the agent has a very high probability to enforce exchanges that lead it to the equilibrium, avoiding other kinds of transitions;

Tolerance: the agent has a high probability to enforce exchanges that lead it to the equilibrium if its material results are far from that state, but it accepts other kinds of transitions.

Table 1 presents a pattern of the probability distribution $\Pi\left(E_{h}\right)$, considering individual agent transitions, characterizing egoist/altruist and fanatic/tolerant agents. Observe that, for an egoist agent, transitions ending in favorable results $\left(E^{+}\right)$occurs with very high probability, whereas, for an altruist agent, the most probable transitions are those ending in

\footnotetext{
${ }^{2}$ In this paper, we consider just a sample of classes of material results. See [3] for the whole family of classes of a QI-MDP, and the interval-based procedure for determining them.
} 
unfavorable results $\left(E^{-}\right)$. For a fanatic agent, the least probable transitions are those not ending in the terminal state $E^{0}$. In contrast, a tolerant agent accepts transitions to states other than $E^{0}$, although with a low probability.

Table 1: A pattern of the interval-valued probability distribution $\Pi\left(\mathbf{E}_{s}\right)$ for individual transitions

\begin{tabular}{c|ccc|ccc}
\hline \multirow{2}{*}{$\Pi\left(\mathbf{E}_{s}\right)$} & \multicolumn{3}{|c|}{ Egoist agents } & \multicolumn{3}{c}{ Altruist agents } \\
\cline { 2 - 7 } & $E^{0}$ & $E^{+}$ & $E^{-}$ & $E^{0}$ & $E^{+}$ & $E^{-}$ \\
\hline$E^{0}$ & low & very high & very low & low & very low & very high \\
$E^{+}$ & low & very high & very low & low & very low & very high \\
$E^{-}$ & low & very high & very low & low & very low & very high \\
\hline \hline & \multicolumn{3}{|c|}{ Fanatic agents } & \multicolumn{3}{c}{ Tolerant agents } \\
\cline { 2 - 7 }$\Pi\left(\mathbf{E}_{s}\right)$ & $E^{0}$ & $E^{+}$ & $E^{-}$ & $E^{0}$ & $E^{+}$ & $E^{-}$ \\
\hline$E^{0}$ & very high & very low & very low & high & low & low \\
$E^{+}$ & very high & very low & very low & high & low & low \\
$E^{-}$ & very high & very low & very low & high & low & low \\
\hline
\end{tabular}

Table 2 shows parts of sample interval state-transition functions $\mathbf{F}$ for systems composed by (a) two tolerant agents and (b) two egoist agents that always disregard the recommendations. The mark $\mathbf{X}$ indicates that the transition is forbidden according to the adopted social rules (both agents increasing results simultaneously, as explained in Sect. 3.). In (b), the highest probabilities appear in the transitions ending in $\left(E^{+}, E^{+}\right)$, representing increasing results for both agents, or in the states $\left(-, E^{+}\right)$or $\left(E^{+},-\right)$when the transitions to the state $\left(E^{+}, E^{+}\right)$are not allowed. The probability around $100 \%$ in the last line of (b) indicates that the agents refuse to exchange (which would lead both to unfavorable results), remaining in the same state $\left(E^{-}, E^{-}\right)$. This shows that this system presents an absorbent state, $\left(E^{-}, E^{-}\right)$, meaning that the system is not able to leave that state if it reaches it, and so it may never achieve the desired target equilibrium point. In (a), one observes the more uniform behavior of tolerant agents, even though the transitions to the states $\left(E^{0}, E^{0}\right)$, $\left(E^{0},-\right)$ and $\left(-, E^{0}\right)$ being the most probable.

We remark that even if the agents present a certain level of obedience, there may be a great deal of uncertainty about the effects of the supervisor's recommendations. Considering an obedience level around 50\%, the state-transition functions shown in Table 2 become the respective ones shown in Table 3, showing an increase in the probability of the transitions ending in $\left(E^{0}, E^{0}\right)$ and also the absence of an absorbent state.

Since the supervisor has no access to the current state (material results of exchanges), it has to rely on observations of the agents' evaluations of their virtual results (debts (D), credits $(\mathrm{C})$ or null results $(\mathrm{N})$ ). Due to their personality traits, they may present different attitudes concerning such evaluations (see Table 4):

Realism: the agent has a very high probability to proceed to realistic evaluations;

Over-evaluation: the agent has a very high probability to report that it has credits;

Under-evaluation: the agent has a very high probability to report that it has debts. 
Table 2: Parts of transition functions $\mathbf{F}$ for pairs of agents that always disregard recommendations

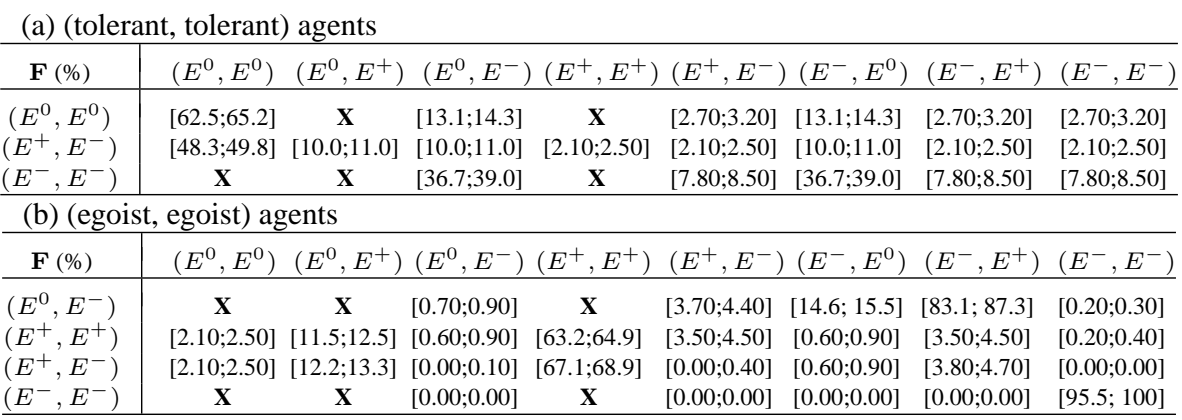

Table 3: Parts of transition functions $\mathbf{F}$ for pair of agents with obedience in $[40 ; 50] \%$

\begin{tabular}{|c|c|c|c|c|c|c|c|c|}
\hline $\mathbf{F}(\%)$ & $\left(E^{0}, E^{0}\right)$ & $\left(E^{0}, E^{+}\right)$ & $\left(E^{0}, E^{-}\right)$ & $\left(E^{+}, E^{+}\right)$ & $\left(E^{+}, E^{-}\right)$ & $\left(E^{-}, E^{0}\right)$ & $\left(E^{-}, E^{+}\right)$ & $\left(E^{-}, E^{-}\right)$ \\
\hline$\left(E^{0}, E^{0}\right)$ & {$[80.1 ; 83.7]$} & $\mathbf{x}$ & {$[6.55 ; 7.15]$} & $\mathbf{x}$ & {$[1.35 ; 1.60]$} & {$[6.55 ; 7.15]$} & {$[1.35 ; 1.60]$} & {$[1.35 ; 1.60]$} \\
\hline$\left(E^{+}, E^{-}\right)$ & {$[73.2 ; 75.6]$} & {$[5.00 ; 5.50]$} & {$[5.00 ; 5.50]$} & {$[1.05 ; 1.25]$} & {$[1.05 ; 1.25]$} & {$[5.00 ; 5.50]$} & {$[1.05 ; 1.25]$} & {$[1.05 ; 1.25]$} \\
\hline$\left(E^{-}, E^{-}\right)$ & $\mathbf{x}$ & $\mathbf{x}$ & {$[18.3 ; 19.5]$} & $\mathbf{x}$ & {$[27.9 ; 30.5]$} & {$[18.3 ; 19.5]$} & {$[27.9 ; 30.5]$} & [27.9;30.5] \\
\hline \multicolumn{9}{|c|}{ (b) (egoist, egoist) agents } \\
\hline $\mathbf{F}(\%)$ & $\left(E^{0}, E^{0}\right)$ & $\left(E^{0}, E^{+}\right)$ & $\left(E^{0}\right.$ & 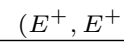 & (2) & (I & (L & $\left(E^{-}, E^{-}\right)$ \\
\hline$\left(E^{0}, E^{-}\right)$ & $\mathbf{x}$ & $\mathbf{x}$ & {$[0.70 ; 0.90]$} & $\mathbf{X}$ & {$[23.1 ; 27.5]$} & {$[7.30 ; 7.75]$} & {$[65.9 ; 69.4]$} & {$[0.20 ; 0.30]$} \\
\hline$\left(E^{+}, E^{+}\right)$ & {$[48.70 ; 58.1]$} & {$[5.75 ; 6.25]$} & {$[0.30 ; 0.45]$} & [31.6;32.5] & {$[1.75 ; 2.55]$} & {$[0.30 ; 0.45]$} & {$[1.75 ; 2.55]$} & {$[0.10 ; 0.20]$} \\
\hline$\left(E^{+}, E^{-}\right)$ & {$[48.70 ; 58.1$} & {$[6.10 ; 6.65]$} & {$[0.00 ; 0.10]$} & {$[33.5 ; 34.5]$} & {$[0.00 ; 0.40]$} & {$[0.30 ; 0.45]$} & {$[1.90 ; 2.35]$} & {$[0.00 ; 0.00]$} \\
\hline$\left(E^{-}, E^{-}\right)$ & $\mathbf{X}$ & $\mathbf{x}$ & {$[0.00 ; 0.00]$} & $\mathbf{x}$ & {$[24.0 ; 25.0]$} & {$[0.00 ; 0.00]$} & {$[24.0 ; 25.0]$} & {$[49.0 ; 50.0]$} \\
\hline
\end{tabular}

\section{Reasoning About Exchanges}

To be able to reason about exchanges between pairs of non-transparent personality-based agents, the supervisor uses Interval-valued Hidden Markov Models (I-HMM) [11].

Definition 5.1. An Interval-valued Hidden Markov Model for exchanges between nontransparent personality-based agents is a tuple $\left\langle\mathbf{E}_{s}, \mathbf{O}, \pi, \mathbf{F}, \mathbf{G}\right\rangle$, where:

(i) the set $\mathbf{E}_{s}$ of states is given by the pairs of classes of material results, where $s$ is the equilibrium point: $\quad \mathbf{E}_{s}=\left\{\left(E^{0}, E^{0}\right),\left(E^{0}, E^{+}\right),\left(E^{0}, E^{-}\right),\left(E^{+}, E^{0}\right),\left(E^{+}, E^{+}\right),\left(E^{+}, E^{-}\right)\right.$, $\left.\left(E^{-}, E^{0}\right),\left(E^{-}, E^{+}\right),\left(E^{-}, E^{-}\right)\right\}$;

(ii) the set $\mathbf{O}$ of observations is given by the possible pairs of agents' evaluations of virtual results: $\mathbf{O}=\{(N, N),(N, D),(N, C),(D, N),(D, D),(D, C),(C, N),(C, D),(C, C)\}$;

(iii) $\pi$ is the initial interval-valued probability distribution over the set of states $\mathbf{E}_{s}$;

(iv) $\mathbf{F}: \mathbf{E}_{s} \rightarrow \Pi\left(\mathbf{E}_{s}\right)$ is the interval state-transition function, which gives, for each state, an interval-valued probability distribution over the set of states $\mathbf{E}_{s}$;

(v) $\mathbf{G}: \mathbf{E}_{s} \rightarrow \Pi(\mathbf{O})$ is the interval observation function that gives, for each state, an interval-valued probability distribution over the set of observations $\mathbf{O}$. 
Table 4: A pattern of the interval-valued probability distribution $\Pi(\mathbf{O})$ over the observations $\mathbf{O}=\{N, D, C\}$ of agents' evaluations of virtual results, in each state

\begin{tabular}{c|ccc|ccc|ccc}
\hline \multirow{2}{*}{$\Pi(\mathbf{O})$} & \multicolumn{3}{|c|}{ Realistic agents } & \multicolumn{3}{c|}{ Over-evaluator agents } & \multicolumn{3}{c}{ Under-evaluator agents } \\
\cline { 2 - 9 } & $\mathrm{D}$ & $\mathrm{N}$ & $\mathrm{C}$ & $\mathrm{D}$ & $\mathrm{N}$ & $\mathrm{C}$ & $\mathrm{D}$ & $\mathrm{N}$ & $\mathrm{C}$ \\
\hline$E^{0}$ & very low & very high & very low & very low & low & very high & very high & low & very low \\
$E^{+}$ & very high & very low & very low & low & medium & high & very high & very low & very low \\
$E^{-}$ & very low & very low & very high & very low & very low & very high & high & medium & low \\
\hline
\end{tabular}

This model allows the supervisor to perform the following HMM tasks [10]:

Task 1: to find the interval-valued probability of a sequence of agents' evaluations of virtual results;

Task 2: to find the most probable sequence of states associated to a sequence of agents' evaluations of virtual results;

Task 3: to maintain an adequate model of agent personality traits, given their observable behaviors: the supervisor adjusts the parameters of its current model to the interval-valued probability of a frequent sequence of observations, in order to compare it with the known models and to classify it.

Whenever a new non-transparent agent join the society, the supervisor assumes the position of an observer, building a I-HHM in order to obtain an adequate model of the personality traits of such agent and to find the most probable state of the system at a given instant. After that, it is able to start making recommendations. We assume that obtaining the model of an agent's personality traits is independent of the agent's degree of obedience. Of course, to discover an agent's degree of obedience is a trivial task.

\section{Simulation Results}

Some simulation results were chosen for discussion, considering the supervisor's tasks detailed in Sect. 5.. For that, interval versions of the dynamic programming algorithms backward-forward (for task 1), Viterbi (for task 2) and Baum-Welch (for task 3) (see [11]), were incorporated in the supervisor behavior (Fig. 1, I-HMM module). The implementation was done in Python, using the module PyInterval [6] for Interval Mathematics.

\subsection{Simulation of Tasks 1 and 2}

The methodology used for the analysis of the performance of the algorithms in tasks $\mathbf{1}$ and 2 considered: (i) test-situations with two agents, combining all different personality traits; (ii) a uniform initial interval-valued probability distribution $\pi$ over the set of states; (iii) the computation of the interval-valued probabilities of occurrence of all sequences of two consecutive observations (agents' evaluations); (iv) the computation of the most probable sequence of states that generates each observation.

Table 5(a) presents some peculiar results obtained for a pair of tolerant/realist agents. As expected, the simulations showed that the observations reflected the actual state transitions. The most probable sequences of observations were those ending in null virtual results, associated to transitions toward the equilibrium (e.g., obs. 1, 2, 3). The transitions that 
did not faithfully reflect the observations were those that took the place of transitions that are forbidden, according to the social rules of the modelling. For example, the transition found for observation 4 (which presented the lowest occurrence probability, for sequences ending in null results) was found in place of $\left(E^{0}, E^{-}\right) \rightarrow\left(E^{0}, E^{0}\right)$, since the latter is a forbidden transition. Observations with very low probability were associated, in general, to transitions that went away from the equilibrium (e.g., obs. 5).

Table 5(b) shows some selected results for a pair of (tolerant/under-evaluator, tolerant/ over-evaluator) agents. As expected, the transitions did not always reflect the observations (e.g., obs. 1, 2). Nonetheless, the overall set of simulations showed that almost $70 \%$ of the observations ending in null results coincided with transitions ending in the equilibrium. However, those observations presented very low probability (e.g., obs. 1 and 3, the latter having the lowest occurrence probability, since it reflected an adequate transition, which was not expected for non realist agents). Observation 4 presented the highest probability, and its associated transition towards to the equilibrium point was the most expected one for a pair of tolerant agents. There was always a high probability that the agents evaluated their virtual results as $(D, C)$ if they were in the equilibrium state, as expected. In general, sequences of observations containing $(D, C)$ were the most probable, whereas sequences of observation presenting $(C, D)$ had almost no probability of occurrence (e.g., obs. 5).

Table 5: Simulation results for pair of agents

\begin{tabular}{cccc}
\hline \multicolumn{4}{c}{ (a) (tolerant/realist, tolerant/realist) } \\
\hline $\mathrm{N}$ & Observation & Probab. (\%) & Probable State Transition \\
\hline 1 & (N,N)-(N,N) & {$[3.32 ; 3.76]$} & $\left(E^{0}, E^{0}\right) \rightarrow\left(E^{0}, E^{0}\right)$ \\
2 & $(\mathrm{D}, \mathrm{D})-(\mathrm{N}, \mathrm{N})$ & {$[3.20 ; 3.63]$} & $\left(E^{+}, E^{+}\right) \rightarrow\left(E^{0}, E^{0}\right)$ \\
3 & $(\mathrm{D}, \mathrm{N})-(\mathrm{N}, \mathrm{N})$ & {$[3.06 ; 3.48]$} & $\left(E^{+}, E^{0}\right) \rightarrow\left(E^{0}, E^{0}\right)$ \\
4 & $(\mathrm{~N}, \mathrm{C})-(\mathrm{N}, \mathrm{N})$ & {$[1.37 ; 1.67]$} & $\left(E^{0}, E^{0}\right) \rightarrow\left(E^{0}, E^{0}\right)$ \\
5 & $(\mathrm{D}, \mathrm{N})-(\mathrm{D}, \mathrm{D})$ & {$[0.33 ; 0.42]$} & $\left(E^{+}, E^{0}\right) \rightarrow\left(E^{+}, E^{+}\right)$ \\
\hline
\end{tabular}

(b) (tolerant/under-evaluator, tolerant/over-evaluator)

\begin{tabular}{cccc}
\hline $\mathrm{N}$ & Observation & Probab. (\%) & Probable State Transition \\
\hline 1 & (N,N)-(N,N) & {$[0.074 ; 0.096]$} & $\left(E^{-}, E^{+}\right) \rightarrow\left(E^{0}, E^{0}\right)$ \\
2 & $(\mathrm{D}, \mathrm{C})-(\mathrm{N}, \mathrm{N})$ & {$[1.745 ; 2.084]$} & $\left(E^{-}, E^{-}\right) \rightarrow\left(E^{-}, E^{0}\right)$ \\
3 & $(\mathrm{C}, \mathrm{D})-(\mathrm{N}, \mathrm{N})$ & {$[0.00 ; 0.0017]$} & $\left(E^{-}, E^{+}\right) \rightarrow\left(E^{0}, E^{0}\right)$ \\
4 & $(\mathrm{D}, \mathrm{C})-(\mathrm{D}, \mathrm{C})$ & {$[33.37 ; 37.04]$} & $\left(E^{+}, E^{-}\right) \rightarrow\left(E^{0}, E^{0}\right)$ \\
5 & $(\mathrm{C}, \mathrm{D})-(\mathrm{C}, \mathrm{D})$ & {$[3.0 ; 7.0] .10^{-6}$} & $\left(E^{-}, E^{+}\right) \rightarrow\left(E^{-}, E^{+}\right)$ \\
\hline
\end{tabular}

\subsection{Simulation of Task 3}

The methodology used for the analysis of the performance of the equilibrium supervisor in the task $\mathbf{3}$ considered the following steps:

1. Given a frequently noticed sequence of observations of evaluations of virtual results, the I-HMM is adjusted by generating new parameters (initial interval distribution, transition and emission interval matrices) for the interval-valued probability of such observations. 
2. The new I-HMM is compared with the models known by the supervisor, stored in a library, using the following procedure. Let $M$ be any parameter of any reference I-HMM (a square interval matrix) and $N$ be the respective parameter of the new I-HMM (an interval matrix of the same dimension of $M$ ). The difference between the new I-HMM and each of such reference models is evaluated by using a kind of distance between the interval matrices $M$ and $N$, given by $\operatorname{dist}(M, N)=\max _{X_{i j} \in M, Y_{i j} \in N} d\left(X_{i j}, Y_{i j}\right)$, where $d\left(\left[x_{1}, x_{2}\right],\left[y_{1}, y_{2}\right]\right)=\max \left\{\left|x_{1}-y_{1}\right|,\left|x_{2}-y_{2}\right|\right\}$ is the distance between intervals $X, Y$. 3. The new I-HMM is then classified either as describing a new model of personality traits or as being of one of the kinds of models maintained in the library, according to a given maximum admissible distance, denoted by $m d$. If the distances between the new I-HMM and all the other models in the library are larger than $m d$, then new I-HMM is classified as a new model, otherwise it is identified as the model from which it takes the least distance.

To adjust the parameters of a given model, we used an interval version of Baum Welch algorithm (which we noticed happened to preserve the compliance of the interval transition matrices to the exchange rules). Table 6 shows the analysis done by the supervisor when observing the interactions between five non-transparent agents with transparent agents. The results were obtained by comparing adjusted I-HMM's (for probabilities of observations) with the other models of pairs of agents, considering $m d=0.7$. For simplicity, only realist agents were considered. ${ }^{3}$

Table 6: Recognition of new personality traits

\begin{tabular}{cccc}
\hline $\mathrm{N}$ & Observation & Prob.(\%) & Personality Traits \\
\hline 1 & (D,D)-(N,N)-(N,N) & {$[75 ; 85]$} & tolerance \\
2 & (D,D)-(N,N)-(N,N) & {$[95 ; 100]$} & new classification \\
3 & (D,N)-(D,D)-(D,D) & {$[55 ; 65]$} & egoism \\
4 & (N,N)-(C,C)-(C,C) & {$[35 ; 45]$} & altruism \\
5 & (D,C)-(C,N)-(D,C)-(C,N) & {$[45 ; 55]$} & new classification \\
\hline
\end{tabular}

For the observation in line 1 (probability around $80 \%$, in interactions with tolerant agents), the least error between the new model and all other models resulted in its compatibility with a model of tolerant agents. So, the supervisor classified the non-transparent agent as tolerant. For the observation in line 5 (probability around $50 \%$, in interactions with tolerant agents), the least error found was larger than the admissible error, and then the supervisor concluded that the agent had a new personality trait. Line 2 shows the dependence of the results on the probability of the observation: if in line 1 it was around $100 \%$, the supervisor would conclude that the agent presented a new personality trait.

\section{Conclusion}

The leads toward the idea of modelling agents' personality traits in social exchange regulation mechanisms, using Interval Mathematics to represent the uncertain knowledge

\footnotetext{
${ }^{3}$ We considered $m d=0.7$ since in the simulations we performed it was empirically observed that models with distances larger than 0.7 no longer behaved as expected for a given personality trait.
} 
about the transitions probabilities that characterize agents' personality-based behaviors and Interval-valued HMM for reasoning about personality-based exchanges. The main contribution of the paper is to provide a reliable tool to deal with the uncertainty that is inherent to the problem of obtaining the characterization of different personalities, due to the specialists divergence in the definition of the various transition probabilities.

Future work is concerning with the fuzzy modelling for the personality-based evaluation of the services that generate qualitative exchange values, in order to consider the different external aspects that may influence the agents' evaluation of a social exchange (e.g., quality, punctuality of services), as proposed in [12].

\section{Acknowledgments}

This is was partially supported by CAPES, FAPERGS, CNPq (Proc. 473201/07-0). The authors are grateful to the referees for their valuable comments.

Resumo. Este trabalho apresenta uma aplicação de Modelos Ocultos de Markov Intervalares para a a modelagem de traços de personalidades de agentes em sistemas multiagentes. Os comportamentos dos agentes são modelados como funções de transição probabilísticas, onde probabilidades intervalares são utilizadas para expressar a incerteza de especialistas na determinação exata dessas probabilidades. O modelo de regulação de trocas sociais baseia-se no conceito de supervisor de equilíbrio, que é capaz de gerar recomendações das melhores trocas que os agentes podem executar para promover o equilíbrio do sistema.

\section{References}

[1] C. Castelfranchi, Engineering social order, in "Engineering Societies in the Agents World" (A. Omicini et al., eds.), pp. 1-18, Springer, Berlin, 2000.

[2] C. Castelfranchi, F. Rosis, R. Falcone, S. Pizzutilo, Personality traits and social attitudes in multiagent cooperation, Applied Artificial Intelligence, 12 (1998), 649-675.

[3] G.P. Dimuro, A.C.R. Costa, Interval-based Markov Decision Processes for Regulating Interactions Between Two Agents in Multi-Agent Systems, in "Applied Parallel Computing", LNCS, n. 3732, pp. 102-111, Springer, Berlin, 2006.

[4] G.P. Dimuro, A.C.R. Costa, L.V. Gonçalves, A. Hübner, Centralized regulation of social exchanges between personality-based agents, in "Coordination, Organizations, Institutions and Norms in MAS II", LNAI, n. 4386, Springer, Berlin, 2007.

[5] G.P. Dimuro, A.C.R. Costa, L.A.M. Palazzo, Systems of exchange values as tools for multi-agent organizations, Journal of the Brazilian Computer Society, 11, No. 1 (2005), 31-50.

[6] P.S. Grigoletti, G.P. Dimuro, L.V. Barboza, Módulo Python para Matemática Intervalar, in "Seleta do XXIX CNMAC" (C.F. Bracciali, L.M. Carvalho, A. Castelo, A. J. Silva Neto, eds.), TEMA - Tendências em Matemática Aplicada e Computacional, Vol. 8, No. 1, pp. 73-82, SBMAC, 2007.

[7] R.E. Moore, "Methods and Applications of Interval Analysis", SIAM, Philadelphia, 1979. 
[8] J. Piaget, "Sociological Studies”, Routlege, London, 1995.

[9] M.L. Puterman, “Markov Decision Processes”, Wiley, New York, 1994.

[10] L.R. Rabiner, A tutorial on Hidden Markov Models and selected applications in speech recognition, Proceedings of the IEEE 77, No. 2 (1989), 257-286.

[11] A.V. Santos, G.P. Dimuro, L.V. Barboza, A.C.R. Costa, R.H.S. Reiser, M.A. Campos, Probabilidades intervalares em modelos ocultos de Markov, in "Seleta do XXVIII CNMAC" (C.F. Bracciali, V.L.R. Lopes, A.J. Silva Neto, A. Sri Ranga, H.M. Yang, eds.), TEMA - Tendências em Matemática Aplicada e Computacional, Vol. 7, No. 2, pp. 361-370, SBMAC, 2006.

[12] A.V. Santos, G.P. Dimuro, B.C. Bedregal, Avaliação fuzzy de trocas sociais entre agentes com personalidades, in "Anais do XXXI CNMAC", Belém, 2008.

[13] P. Walley, Measures of uncertainty, Artificial Intelligence, 83 (1996), 1-58.

[14] M. Wooldridge, “An Introduction to Multi-Agent Systems”, Wiley, New York, 2002. 